


\title{
A influência dos fundos de investimentos na formação do preço do trigo na Bolsa de Cereais de Chicago
}

\author{
The influence of investiment funds in \\ the formation of wheat price in the \\ Chicago Board of Trade
}

\author{
Argemiro Luís Brum* | Daniel Knebel Baggio** | Francisca Mendonça de \\ Souza*** Daniel Claudy da Silveira**** | Isoé Nicolas Schneider***** \\ http://dx.doi.org/10.51861/ded.dmvu.2.003 \\ Recebido em 18 de novembro de 2020. Aceito em 16 de junho de 2021.
}

\begin{abstract}
Resumo
Este artigo analisa a influência dos fundos na formação do preço futuro do trigo junto à Bolsa de Cereais de Chicago (CBOT). O objetivo principal é verificar em que níveis tais Fundos influenciam na formação de seus preços, e como isso ocorre, tomando-se o período de tempo entre 2006 a 2020. As análises são realizadas de correlações e regressões entre as variáveis preço futuro do trigo na $C B O T$ e os diversos grupos de traders que negociam na Bolsa. Dentre os resultados obtidos, notou-se que cerca de $32 \%$ da cotação do trigo na CBOT seria explicado pela atuação conjunta dos fundos de investimento, dos especuladores e dos chamados negociadores que não precisam declarar as suas negociações, enquanto outros $25 \%$ são influenciados pela atuação dos investidores comerciais. Os resultados contribuem com achados empíricos relevantes que destacam a importância do movimento especulativo na formação de preços dos cereais e particularmente do trigo na CBOT, o qual repercute sobre os preços pagos aos produtores do cereal no Brasil.
\end{abstract}

Palavras-chave: Fundos de investimento; Trigo; Bolsa de Chicago; Cotações; Correlação.

\begin{abstract}
This article analyzes the influence of funds on the formation of the price of wheat with the Chicago Cereals Exchange (CBOT). The main objective is to verify at what levels such Funds influence the formation of their prices, and how this occurs, taking the time period between 2006 to 2020. The analyzes are carried out of correlations and regressions between the variables future price of wheat in the CBOT and the various groups of traders who trade on the exchange. Among the results obtained, it was noted that about $32 \%$ of the price of wheat in CBOT would be explained by the joint performance of investment funds, speculators and so-called negotiators who do not need to declare their negotiations, while another $25 \%$ are influenced by commercial investors. The results contribute to relevant empirical findings that highlight the importance of the speculative movement in the formation of prices of cereals and particularly of wheat at $C B O T$, which affects the prices paid to cereal producers in Brazil.
\end{abstract}

Keywords: Investment funds; Wheat; Chicago Stock Exchange; Quotations; Correlation.

\footnotetext{
* Professor titular junto ao PPGDR da UNIJUI, doutor em Economia Internacional pela EHESS de Paris (França). Endereço: Rua do Comércio, n³000, Bairro Universitário, CEP 98700-000 - ljuí, RS.

ORCID: https://orcid.org/0000-0002-8763-9514. E-mail: argelbrum@unijui.edu.br.

** Professor titular junto ao PPGDR da UNIJUÍ, doutor em Contabilidade e Finanças pela Universidade de Zaragoza-Espanha, e Professor do Programa de Gestão Estratégica de Organização/URI. Endereço: Rua do Comércio, $n^{\circ}$ 3000, Bairro Universitário, CEP 98700-000 - ljuí, RS.

ORCID: https://orcid.org/0000-0002-6167-2682. E-mail: danibaggio@gmail.com.

*** Professora de Pós-Graduação na UNIJUÍ, doutora em Estatística e Análise de Dados - Econometria pelo Instituto Universitário de Lisboa (ISCTE-IUL) - Portugal, Investigadora da Universidade da Beira Interior -UBI/Portugal. Endereço: Rua do Comércio, n³000, Bairro Universitário, CEP 98700-000 - ljuí, RS.

ORCID: https://orcid.org/0000-0003-3011-0086. E-mail: kikamatcom@gmail.com.

**** Professor de Economia da UNIJUI, doutor em Desenvolvimento Regional pela Universidade de Santa Cruz do Sul (UNISC), e Professor do Programa de Gestão Estratégica de Organização/URI Santo Ângelo. Endereço: Rua do Comércio, n³ 3000, Bairro Universitário, CEP 98700-000 - ljuí, RS.

ORCID: https://orcid.org/0000-0003-4379-6144. E-mail: daniel.silveira@unijui.edu.br.

***** Mestre em Desenvolvimento Regional pela UNIJUÍ, doutorando pela Universidad de Zaragoza (Espanha). Endereço: Rua do Comércio, n³000, Bairro Universitário, CEP 98700-000 - ljuí, RS.

ORCID: https://orcid.org/0000-0002-2806-5135.E-mail: iso.nicolas@hotmail.com.
} 


\section{INTRODUÇÃO}

A cultura do trigo é uma das mais importantes atividades econômicas do setor agropecuário mundial. Sua produção vem crescendo anualmente, sendo que apenas entre 2018/19 e 2020/21 a mesma deverá receber um incremento de $5,4 \%$ ou 39,5 milhôes de toneladas em termos globais. O comércio mundial deste cereal cresce ainda mais rápido, devendo atingir um aumento de 7,9\% no mesmo período, para se estabelecer, em termos médios, em 185,8 milhôes de toneladas em 2020/21.

Assim como as principais commodities negociadas no mercado mundial, o trigo igualmente tem sua comercialização balizada pelo comportamento de suas cotaçốes em bolsa de mercadorias. No caso, na Bolsa de Cereais de Chicago (CBOT), nos EUA.

E, assim como as demais commodities, o cereal também vem assistindo mudanças importantes em sua estrutura de formação de preços na bolsa nas últimas décadas. De uma realidade mercadológica centrada na oferta, demanda, estoques, clima e políticas públicas, suas cotações em Chicago passaram a sofrer influência dos movimentos de compra e venda dos Fundos de Investimento. Tais movimentos, em quase sua totalidade meramente especulativos, embora importantes para oferecer liquidez aos negócios bursáteis, cada vez menos levam em consideração as questóes clássicas inerentes ao mercado. Esta realidade influencia diretamente a formação dos preços junto aos produtores rurais do cereal em todo o mundo, dificultando a compreensão das tendências de mercado e a tomada de decisão de venda do cereal.

Neste contexto, o presente trabalho visa analisar a influência do movimento destes Fundos na compra e venda de contratos de trigo na $C B O T$ e, por conseguinte, no estabelecimento de sua cotação diária. Estudos anteriores focaram na relação de outras commodities e os movimentos especulativos dos fundos e investimentos (BRUM et al., 2019a; BRUM et al., 2019b; BRUM et al., 2019c; BRUM et al., 2020; SOUZA et al., 2020; CORREIA \& LIMA, 2020; SILVEIRA et al., 2019) sobre os mercados. Além disso, destacam-se os seguintes estudos que estudaram o impacto de determinadas variáveis nos preços futuros das commodities (COUTINHO FILHO, 2018; GALVÃO, 2015; HERREROS, BARROS \& BENTES (2010); LISZBINSKI, 2020; MARGARIDO et al., 2014; SOUZA et al., 2020).

O objetivo central é detectar em que dimensão a ação comercial dos Fundos influencia na formação das cotaçốes do trigo em Chicago em relação aos fatores clássicos presentes no mercado. Metodologicamente, os dados foram organizados e processados no software SPSS 22.00, sendo que para a análise e interpretação dos mesmos, inicialmente realizou-se a construção de gráficos para análise da evolução do preço do trigo e da quantidade de contratos negociados 
pelos diferentes grupos investidores, resultando em uma análise descritiva e preliminar dos dados, semelhante à realizada no estudo de Herreros, Barros e Bentes (2010). Destaca-se a importância de estudar os fundos e investimentos e a sua influência na formação dos preços futuros das commodities agrícolas e na formação de poupança dos cidadãos (BAGGIO et al., 2007; BAGGIO et al., 2010; BAGGIO et al., 2012; BAGGIO et al., 2018; FERRUZ et al., 2019; SCHNEIDER et al., 2019, BAGGIO, et al., 2019).

Em um segundo momento passou-se para a análise inferencial dos dados, a partir de análises de correlação e de regressão múltipla. Inicialmente realizou-se o coeficiente de correlação de Pearson ( $r$ ) para verificar a associação entre as variáveis estudadas. O coeficiente de correlação de Pearson (r) mede o grau da correlação linear entre duas variáveis quantitativas. É um índice adimensional com valores situados entre $-1,0$ e 1,0 inclusive, que reflete a intensidade de uma relação linear entre dois conjuntos de dados (LIRA, 2004; FIGUEIREDO FILHO \& SILVA JÚNIOR, 2009). Enfim, realizouse a análise de regressão múltipla, utilizando-se a variável preço futuro do trigo como dependente e todas as demais como variáveis independentes, visando verificar o poder explicativo das demais variáveis sobre a formação do preço futuro do cereal.

O presente artigo está dividido em três partes além desta introdução e das consideraçóes finais. A primeira parte destaca uma breve análise da economia do trigo no mundo e no Brasil. Na segunda parte tem-se, de forma integral, a metodologia utilizada para se obter as respostas ao problema posto e o objetivo central, assim como se apresenta as hipóteses levantadas. Na terceira parte, são apresentados os resultados obtidos com o estudo.

\section{A ECONOMIA DO TRIGO: ASPECTOS MUNDIAIS E NO BRASIL}

O trigo é uma cultura milenar, estando presente na alimentação humana pelo menos quatro mil anos antes de Cristo. Seu cultivo teria iniciado na Mesopotâmia, na região chamada de Crescente Fértil, área hoje compreendida entre o Egito e o Iraque. Rapidamente, com o avanço dos deslocamentos humanos pelo Planeta, o cereal foi acompanhando este movimento e se espalhou pelo mundo. Hoje é uma cultura imprescindível na alimentação humana, porém, muitas vezes igualmente é utilizado na composição de rações, para a alimentação animal.

A produção mundial de trigo passa a crescer rapidamente no século XX, na esteira da modernização agrícola que se inicia pelos EUA. Através dela a produtividade do cereal aumenta e as sementes são adaptadas a novas regióes de produção. 
Nesta safra 2020/21 a produção global de trigo está sendo projetada em 770,5 milhões de toneladas. Os maiores produtores mundiais são a União Europeia (conjunto de 27 países europeus) e a China, ambos com 136 milhões de toneladas anuais cada um. Após, tem-se a Rússia com 78 milhões, os EUA com 50 milhões, o Canadá com 36 milhões, a Austrália com 28,5 milhóes e a Ucrânia com 27 milhóes de toneladas. Na América do Sul, a Argentina é o grande produtor com projeção de gerar 19,5 milhões de toneladas em 2020/21 (USDA, 2020).

O comércio mundial de trigo chega, em 2020, a 188 milhóes de toneladas. Ou seja, do total mundial produzido, se comercializa entre as naçóes cerca de $24 \%$. Dito de outra forma, $76 \%$ da produção mundial de trigo é consumida e/ou estocada no interior dos países produtores, enquanto outros $24 \%$ fazem parte do comércio mundial do cereal. Os maiores exportadores de trigo no mercado mundial são: Rússia, com 37,5 milhões de toneladas projetadas para o corrente ano comercial (2020/21); EUA com 26,5 milhóes; União Europeia com 25,5 milhões; Canadá com 25 milhões; Austrália com 19 milhôes; e Ucrânia com 18 milhões de toneladas. A Argentina igualmente é um importante exportador mundial, devendo alcançar 13,5 milhóes de toneladas exportadas nesta temporada (USDA, 2020).

Já os principais importadores presentes no mercado mundial são especialmente países do mundo subdesenvolvido. Neste grupo, tem-se a África do Norte, com 29,6 milhóes de toneladas, com o Egito sendo o maior importador individual do cereal (ao redor de 13 milhóes de toneladas anuais), seguida do Sudeste Asiático com projeção de importar 26,4 milhóes de toneladas nesta temporada. Em terceiro lugar tem-se o Oriente Médio, com 18 milhões de toneladas. Individualmente, ainda se destacam cinco países no mercado importador: a China, com 7 milhóes de toneladas; o Brasil com 6,7 milhões; Bangladesh com 6,6 milhões; Japão com 5,6 milhões; e a Nigéria com 5,1 milhões de toneladas (USDA, 2020).

Neste contexto, a projeção para o ano comercial 2020/21, em termos mundiais, é de uma produção total de 770,5 milhões de toneladas, um consumo sob forma de ração animal ao redor de 134,6 milhóes de toneladas, e o consumo humano em 616,3 milhóes. Considerando que o ano inicia com estoques de passagem de 300 milhóes de toneladas, sobram em estoque final (passagem para 2021/22) o total de 319,6 milhóes de toneladas.

Por sua vez, os preços internacionais do trigo têm, igualmente, na Bolsa de Cereais de Chicago (CBOT) sua referência principal. Na mesma, a cotação para o primeiro mês, em meados de setembro de 2020, estava ao redor de US\$ 5,45/bushel, sendo que um bushel de trigo equivale a 27,21 quilos. Ao câmbio daquele momento, o equivalente a um saco de 60 quilos (medida 
usada no Brasil) valia $\mathrm{R} \$ 63,45$. Como já analisado em artigos anteriores (BAGGIO et al. 2019 e SOUZA, et al., 2020; BRUM et al. 2019a; BRUM et al. 2019b; BRUM et al. 2019c e BRUM et al. 2020) também neste mercado, desde meados dos anos de 1990, os fundos de investimento passaram a atuar de forma importante, em caráter especulativo, gerando alterações na características do mercado, pois a lógica comercial sustentada pela oferta, demanda, estoques e clima nas regiốes de produção perde espaço na explicação do comportamento das cotações, sendo substituída em boa parte pela ação especulativa destes fundos. Tema este que será o objeto central deste artigo.

No Brasil, o trigo chegou com a colonização portuguesa, ainda no início do século XVI. Sua produção sempre foi, basicamente, para o consumo humano, sendo o cereal o produto central por onde se consolidou a chamada modernização agrícola brasileira, no bojo da Revolução Verde, a partir da década de 1950 (BRUM, 1988).

Apesar dos esforços do Estado brasileiro durante o auge desta modernização (1950 a 1980), o Brasil jamais conseguiu a autossuficiência em trigo, acabando por se tornar um dos maiores importadores individuais do cereal desde então.

Os dois maiores Estados produtores de trigo no Brasil são o Paraná e o Rio Grande do Sul. Os dois, somados, representam $88 \%$ da produção prevista para 2020. Esta participação já foi maior, tendo atingido a 90,6\% em 2011. Isto representa, nas projeçóes para 2020, um total de 3,28 milhões de toneladas no Paraná e 2,7 milhões no Rio Grande do Sul, sobre um total esperado no Brasil de 6,81 milhôes de toneladas.

A partir de 1950, portanto, a área semeada com trigo no Brasil, impulsionada pelos programas de apoios estatais, avançou significativamente, atingindo seu auge em 1986, com 3,9 milhôes de hectares. A produtividade média nacional nunca foi significativa neste período, tendo atingido seu máximo em 1987 com 1.786 quilos/hectare (29,8 sacos/hectare). Esta realidade, somada a preços relativamente baixos pagos aos produtores rurais, aos riscos constantes gerados pelo clima do sul do país e, especialmente, a retirada do apoio oficial à triticultura a partir de 1990, levou a uma redução significativa na área semeada com o cereal a partir de 1987, com a mesma chegando a apenas 1,03 milhão de hectares em 1994. Apenas em 2002 esta área volta a superar novamente os 2 milhóes de hectares (2,05 milhões), evoluindo em alguns momentos até 2,7 milhões de hectares, tendo a safra de 2020 alcançado 2,3 milhóes de hectares semeados. Neste período a contar de 2002, entretanto, o avanço da tecnologia de produção permitiu um aumento importante na produtividade média do cereal, especialmente no sul do país. A mesma chega a atingir 2.736 quilos/hectare (45,6 sacos/hectare) em 2010 
e o recorde de 3.175 quilos/hectare (52,9 sacos/hectare) em 2016, sendo que neste ano, o Paraná registrou 52,3 sacos/hectare e o Rio Grande do Sul 53,6 sacos/hectare. Para a safra 2020 a expectativa é de uma produtividade média de 2.928 quilos/hectare (48,8 sacos/hectare) (CONAB, 2020). Assim, a redução na área semeada é compensada largamente pelo aumento da produtividade média nas duas últimas décadas, fato que mantém a produção brasileira entre 5,5 e 6,5 milhões de toneladas em condiçóes normais de clima.

Tanto é verdade que se o país tivesse mantido a produtividade recorde atingida no período de 1950 a 1998, que foi de 1.786 quilos em 1987, a produção final esperada para 2020 seria de apenas 4,16 milhóes de toneladas, ou seja, $39 \%$ a menos do que o volume realmente projetado.

Dito isso, o país ainda precisa importar anualmente entre 6 a 7 milhóes de toneladas, já que o consumo nacional de trigo gira entre 10,5 e 12,5 milhões de toneladas, dependendo da situação econômica interna. Estas importaçôes vêm particularmente da Argentina, a qual fornece entre 80 a $90 \%$ do trigo que o Brasil compra no exterior anualmente. É neste contexto que as cotaçốes na $C B O T$ impactam na economia do trigo brasileiro, já que a Argentina o exporta baseada nas cotaçóes daquela Bolsa. Além deste, outro elemento fundamental para definir o preço interno do cereal é o câmbio, pois uma desvalorização do Real eleva o preço de importação, implicando, geralmente, em melhor preço ao trigo de qualidade superior produzido no Brasil. Esta realidade está cristalizada, por exemplo, neste ano de 2020 no país.

Todavia, o tema central deste artigo sendo os efeitos da atuação dos fundos de investimento nas cotaçôes do trigo na $C B O T$, a questão cambial e outros elementos que interferem na formação do preço do trigo brasileiro não serão aqui objeto de análise, ficando para um trabalho posterior.

\section{METODOLOGIA}

Para cumprir os objetivos propostos neste estudo os dados foram coletados na base de dados da Commodities Futures Trading Comission (CFTC), correspondentes ao período de 03/01/2006 a 28/07/2020, junto às seguintes categorias de traders: "non-commercial traders", "non-commercial spread traders", "small traders, commercial traders" e os "Commodity Index Traders" (CITs). Além disso, utilizou-se, para o mesmo período, a cotação histórica do trigo na CBOT.

A CFTC classifica os traders em dois grupos: "non-commercial traders" e "commercial traders". A diferença básica entre os dois grupos está relacionada com o nível de influência que cada um exerce sobre a formação de preços futuros, assim como em relação a declaração diária de suas posiçôes mantidas em aberto (open interest). 
O primeiro grupo é composto pelos "non-commercial traders", os "non-commercial spread traders" e os "small traders". Os "non-commercial traders" e os "non-commercial spread traders" são compostos, em grande parte, pelos fundos de hedge e pelos fundos de commodities que, classificados como "large speculators" (grandes especuladores), são obrigados, pelas normas da CFTC, a declarar as posiçóes assumidas nos pregóes diários.

Os "small traders" não precisam declarar suas posições, uma vez que, sendo considerados como pequenos especuladores, a sua participação não tem grande influência na determinação dos preços.

No segundo grupo encontram-se os "commercial traders", que utilizam contratos futuros para realizar hedging visando proteger-se do risco ou prejuízo. Do mesmo modo que os "large speculators", os "commercial traders" são obrigados a declarar suas posiçôes assumidas diariamente.

Além disso, existem os CIT's, os quais compóem suas carteiras por uma cesta de setores importantes da economia mundial, ponderados pela importância que os mesmos representam na economia global. Dentre eles tem-se a energia, os metais industriais, os metais preciosos, a agricultura e a pecuária. A partir de 2006, a CFTC passou a incluir em seus relatórios semanais a informação relacionada a posição assumida pelos CIT's em $12 \mathrm{com}$ modities agrícolas. Anteriormente, essas informaçốes eram incluídas junto aos dos "commercial traders". Entretanto, a partir de reclamaçóes de agentes do mercado e investigações do Congresso Americano sobre transparência das informações que não incluíam as posições especulativas dos fundos de investimento, a CFTC foi forçada a incluir, em seus relatórios semanais, o volume de contratos abertos long (comprados) e short (vendidos) assumidos pelos CIT's (HERREROS, BARROS \& BENTES, 2010). Destaca-se que os registros sobre o trigo passaram a ser feitos, nesta nova modalidade, a partir da data do 03/01/2006 na CFTC. Portanto, o presente artigo utilizou toda a série histórica desta variável.

A CFTC publica os relatórios do Commitments of Traders (COT) para ajudar o público a compreender a dinâmica do mercado. Especificamente, os relatórios do COT fornecem uma análise da participação aberta, a cada terça-feira, para futuros e opçóes em mercados futuros, nos quais 20 ou mais comerciantes detêm posiçôes iguais ou acima dos níveis de relatórios estabelecidos pela CFTC (CFTC, 2019).

A CFTC não identifica as razões específicas para as posições dos comerciantes e, portanto, essa informação não leva em consideração as classificaçôes do trader. Na prática, isso significa, por exemplo, que os dados de posição de um negociante classificado na categoria "produtor/comerciante/ processador/usuário”, para uma mercadoria em particular, incluirão todas 
as suas posições naquela mercadoria, independentemente se a posição é de hedge ou especulação (CFTC, 2019).

Os dados agregados referentes às posiçôes reportadas são publicados pela CFTC em seus relatórios semanais de Compromissos de Comerciantes. Os dados são agregados para proteger a identidade de qualquer comerciante "reportável” individual (CFTC, 2019).

Uma vez que os negociantes frequentemente carregam posições de futuros, através de mais de um corretor, e controlam ou têm interesse financeiro em mais de uma conta, a Comissão obtém rotineiramente informaçóes que permitem agregar contas relacionadas (CFTC, 2019). Assim, para a realização do presente estudo, utilizaram-se as seguintes variáveis:

Quadro 01 - Variáveis utilizadas para o desenvolvimento da pesquisa.

\begin{tabular}{|c|l|l|l|}
\hline Variável & \multicolumn{1}{|c|}{ Nome Original } & \multicolumn{1}{|c|}{ Nome Traduzido } & \multicolumn{1}{|c|}{ Expressão Utilizada } \\
\hline Var1 & $\begin{array}{l}\text { No Commercial Position } \\
\text { Long }\end{array}$ & Não-Comerciais Long & NComm_Positions_Long_All_NoCIT \\
\hline Var2 & $\begin{array}{l}\text { No Commercial Position } \\
\text { Short }\end{array}$ & Não-Comerciais Short & NComm_Positions_Short_All_NoCIT \\
\hline Var3 & $\begin{array}{l}\text { No Commercial Position } \\
\text { Spread }\end{array}$ & $\begin{array}{l}\text { Não-Comerciais } \\
\text { Spread }\end{array}$ & NComm_Postions_Spread_All_NoCIT \\
\hline Var4 & Commercial Position Long & Comericiais Long & Comm_Positions_Long_All_NoCIT \\
\hline Var5 & Commercial Position Short & Comerciais Short & Comm_Positions_Short_All_NoCIT \\
\hline Var6 & No Report Position Long & Não-Declaram Long & NonRept_Positions_Long_All \\
\hline Var7 & No Report Position Short & Não-Declaram Short & NonRept_Positions_Short_All \\
\hline Var8 & CIT Position Long & CIT Long & CIT_Positions_Long_All \\
\hline Var9 & CIT Position Short & CIT Short & CIT_Positions_Short_All \\
\hline
\end{tabular}

Fonte: elaborado pelos autores, a partir de informações da CFTC.

Além destas variáveis, utilizou-se também o preço futuro do trigo na CBOT como variável. Os dados utilizados referem-se à quantidade de contratos diários negociados, com periodicidade semanal, ou seja, foram utilizadas as quantidades de contratos negociados a cada terça-feira para cada grupo de investidores. Com relação ao preço do trigo, utilizou-se o preço de fechamento do cereal na Bolsa de Cereais de Chicago, correspondente a toda terça-feira de cada semana.

Os dados foram organizados e processados no software SPSS 22.00. Para a análise e interpretação dos dados, inicialmente realizou-se a construção de gráficos para análise da evolução do preço do trigo e da quantidade de contratos negociados pelos diferentes grupos investidores, resultando em uma 
análise descritiva e preliminar dos dados, semelhante à realizada no estudo de Herreros, Barros e Bentes (2010).

Em um segundo momento passou-se para a análise inferencial dos dados, a partir de análises de correlação e de regressão múltipla. Inicialmente realizou-se o coeficiente de correlação de Pearson ( $r$ ) para verificar a associação entre as variáveis estudadas. $\mathrm{O}$ coeficiente de correlação de Pearson (r) mede o grau da correlação linear entre duas variáveis quantitativas. É um índice adimensional com valores situados entre $-1,0$ e 1,0 inclusive, que reflete a intensidade de uma relação linear entre dois conjuntos de dados (LIRA, 2004; FIGUEIREDO FILHO \& SILVA JÚNIOR, 2009). Os intervalos e a intensidade da correlação linear podem ser observados no quadro a seguir.

Quadro 02 - Intervalos de intensidade da correlação linear.

\begin{tabular}{|c|l|}
\hline Intervalo de correlação (+ ou -) & \multicolumn{1}{|c|}{ Interpretação } \\
\hline 0.00 a 0.19 & Correlação bem fraca \\
\hline 0.20 a 0.39 & Correlação fraca \\
\hline 0.40 a 0.69 & Correlação moderada \\
\hline 0.70 a 0.89 & Correlação forte \\
\hline 0.90 a 1.00 & Correlação muito forte \\
\hline
\end{tabular}

Fonte: elaborado pelos autores, adaptado de Callegari-Jacques (2003, p. 90).

Em um terceiro momento realizou-se a análise de regressão múltipla, utilizando-se a variável preço futuro do trigo como dependente e todas as demais como variáveis independentes, visando verificar o poder explicativo das demais variáveis sobre a formação do preço futuro do cereal. A partir da regressão linear foi possível compreender a influência de cada variável no modelo explicativo e, por meio do coeficiente de determinação $\mathrm{R}^{2}$ e do coeficiente de determinação ajustado, indicar quanto o modelo foi capaz de explicar de acordo com os dados utilizados (CAMERON, 1993; SELL, 2005; MONTGOMERY et al., 2006). O $\mathrm{R}^{2}$ poderá variar entre 0 e 1 , indicando, em percentagem, o quanto o modelo consegue explicar os valores observados. Quanto maior o $\mathrm{R}^{2}$, mais explicativo é o modelo (CORRAR, PAULO \& DIAS FILHO, 2009).

Igualmente, realizou-se uma análise de regressão a partir da estimação stepwise, que se refere ao método que possibilita examinar a contribuição adicional de cada variável independente do modelo, pois cada variável é considerada para inclusão antes do desenvolvimento da equação (HAIR JR. et al. 2005; CORRAR, PAULO e DIAS FILHO, 2009). Neste contexto, buscou-se responder as seguintes hipóteses: 
Quadro 03 - Hipóteses a serem verificadas.

\begin{tabular}{|c|l|}
\hline Número & \multicolumn{1}{c|}{ Hipótese } \\
\hline H1: & $\begin{array}{l}\text { Os grupos de investidores "Não Comerciais short" impactam negativamente no preço futuro } \\
\text { do trigo }\end{array}$ \\
\hline H2: & $\begin{array}{l}\text { Os grupos de investidores "Não Comerciais long" impactam positivamente no preço futuro } \\
\text { do trigo }\end{array}$ \\
\hline H3: & Os grupos de investidores "Comerciais long" impactam positivamente no preço futuro do trigo \\
\hline H4: & $\begin{array}{l}\text { Os grupos de investidores "Comerciais short" impactam negativamente no preço futuro do } \\
\text { trigo }\end{array}$ \\
\hline H5: & Os grupos de investidores "CIT short" impactam negativamente no preço futuro do trigo \\
\hline H6: & Os grupos de investidores "CIT long" impactam positivamente no preço futuro do trigo \\
\hline
\end{tabular}

Fonte: elaborado pelos autores.

\section{ANÁLISE E INTERPRETAÇÃO DOS RESULTADOS}

Os resultados do presente estudo são apresentados em dois momentos. Inicialmente uma apresentação da evolução histórica dos preços futuros do trigo, desde o ano de 2006 até finais de julho de 2020 e suas relações com os contratos negociados pelos diferentes grupos de investidores denominados na metodologia. Em um segundo momento se busca analisar, a partir de modelos de regressão, o impacto dos grupos de investidores na formação do preço futuro do trigo.

O gráfico 1 apresenta a evolução do preço do trigo ao longo dos últimos 14 anos. É perceptível que o preço futuro do trigo tem percorrido um horizonte de preço entre US\$3,28 e US\$12,44/bushel. O preço médio alcançado ao longo dos 14 anos foi de USS 5,73/bushel, sendo que nos últimos cinco anos da série histórica o preço oscilou na faixa de US\$ 4,00 a US\$ 6,00, apresentando um movimento lateralista-altista. A tendência do gráfico para o período é lateralista, mesmo que o recorte temporal de maior oscilação dos preços tenha ocorrido entre os anos de 2007 e 2013 . Verifica-se ainda que a partir do ano de 2013 a commodity passou para uma tendência de baixa do seu preço futuro, mesmo que no longo prazo o gráfico apresente uma evolução lateralista do preço.

O forte aumento dos preços do trigo, assim como das demais commodities, entre 2007 e 2008, é reflexo dos efeitos da crise econômico-financeira do período. A mesma provocou uma quebra significativa dos agentes do sistema financeiro mundial, atingindo a economia real. Com isso, os Estados se viram obrigados a injetarem recursos públicos na economia, procurando socorrer, mesmo que parcialmente, tais agentes, assim como a economia de forma geral. Além disso, outra medida de apoio à economia foi a redução das taxas dos juros básicos a praticamente zero, fato que levou os Fundos de Investimento a 
venderem posiçôes nos mercados de títulos públicos e dos títulos do Tesouro, transferindo estes recursos para aplicações significativas nos mercados futuros de commodities, dentre elas o trigo.

Gráfico 1: Evolução das cotações do triqo na CBOT (em US\$/bushel)

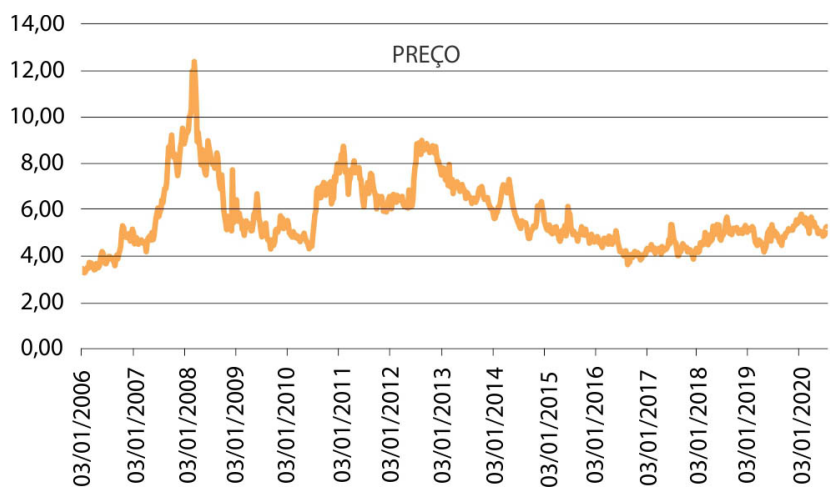

Fonte: elaborado pelos autores, com base nos dados da CBOT.

No momento em que se analisa a relação existente entre os grupos de investidores e o preço futuro do trigo, verifica-se associaçóes positivas e negativas entre eles, como pode ser observado no quadro 04 , contendo os índices de correlações.

Quadro 04: Correlação entre os grupos de investidores e o preço futuro do trigo.

\begin{tabular}{|l|l|l|l|l|l|l|l|l|l|l|}
\hline Variáveis & $\begin{array}{l}\text { Trigo } \\
\text { Futuro }\end{array}$ & NCPL & NCPS & NCPSP & CPL & CPS & NRPL & NRPS & CITPL & CITPS \\
\hline $\begin{array}{l}\text { Trigo } \\
\text { Futuro }\end{array}$ & 1,000 & & & & & & & & & \\
\hline NCPL & 0,019 & 1,000 & & & & & & & & \\
\hline NCPS & $-0,384$ & 0,516 & 1,000 & & & & & & & \\
\hline NCPSP & 0,002 & 0,524 & 0,269 & 1,000 & & & & & & \\
\hline CPL & $-0,314$ & 0,548 & 0,723 & 0,554 & 1,000 & & & & & \\
\hline CPS & $\mathbf{0 , 4 1 9}$ & 0,032 & $-0,592$ & 0,018 & $-0,093$ & 1,000 & & & & \\
\hline NRPL & $-0,052$ & 0,468 & 0,512 & 0,360 & 0,663 & 0,131 & 1,000 & & & \\
\hline NRPS & 0,436 & $-0,142$ & $-0,485$ & $-0,104$ & $-0,176$ & 0,740 & 0,187 & 1,000 & & \\
\hline CITPL & $\mathbf{0 , 4 6 0}$ & $-0,247$ & $-0,480$ & $-0,151$ & $-0,270$ & 0,770 & $-0,037$ & 0,679 & 1,000 & \\
\hline CITPS & 0,095 & 0,204 & 0,082 & 0,579 & 0,255 & $-0,069$ & $-0,006$ & $-0,262$ & 0,109 & 1,000 \\
\hline
\end{tabular}

*Trigo Futuro $=$ Preço do Trigo Futuro; NCPL $=$ NComm_Positions_Long_All_NoCIT; NCPS $=$ NComm_Positions_Short_All_NoCIT; CPL $=$ Comm_ Positions_Long_All_NoCIT; CPS $=$ Comm_Positions_Short_All_NoCIT; NCPSP $=$ NComm_Postions_Spread_All_NoCIT; NRPL $=$ NonRept_Positions_ Long_All; NRPS $=$ NonRept_Positions_Short_All; CITPL $=$ CIT_Positions_Long_All; CITPS $=$ CIT_Positions_Short_All.

Fonte: elaborado pelos autores, a partir de dados da CBOT e da CFTC. 
Destaca-se a associação positiva entre a variável preço futuro do trigo e o grupo de investidores "CIT Positions Long", com um índice de correlação moderado de 0,460 . O mesmo pode ser observado ao longo da evolução do volume de contratos "CIT Positions Long” e o preço futuro do trigo, apresentando um comportamento similar ao longo dos 14 anos estudado (ver gráfico 2). Portanto, é possível verificar que o contrato negociado por este grupo de investidores acompanha o comportamento dos preços futuros do trigo.

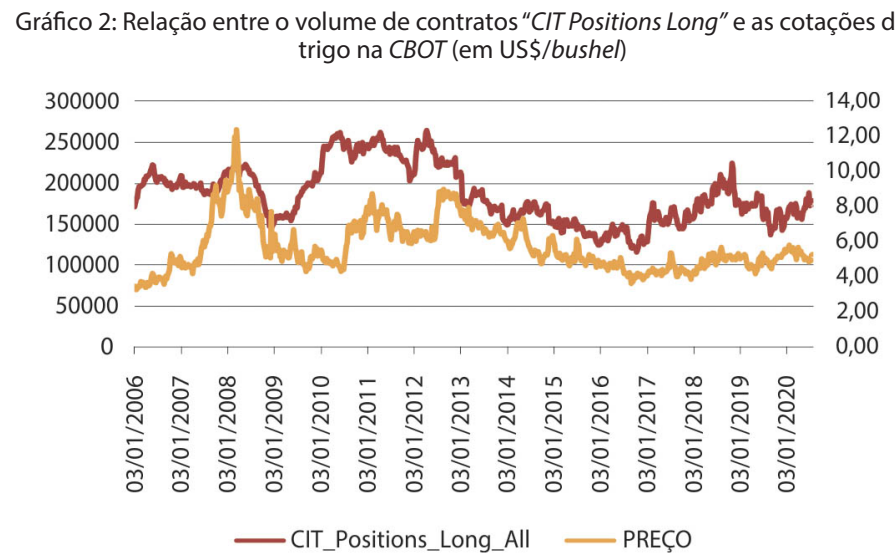

Fonte: elaborado pelos autores, com base nos dados da CBOT e da CFTC.

A mesma associação pode ser observada nas variáveis "NonRept_Positions_Short_All" e "Comm_Positions_Short_All_NoCIT", já que ambos apresentaram índices de correlação positivos e moderados, respectivamente de 0,436 e 0,419 . A relação da evolução do preço futuro do trigo e a variável “NonRept_Positions_Short_All” pode ser observada no gráfico 03.

Com relação as associaçôes negativas, destacam-se as variáveis "No Commercial Position Short" e "Commercial Position Long”, com índices de correlação de $-0,384$ e -0,314, respectivamente, que podem ser verificadas nos gráficos 04 e 05 a seguir.

Importante destacar a evolução histórica dos preços e da quantidade de contratos negociados podem ser observados tanto no gráfico 4 (anterior) e no gráfico 5 (a seguir). 
Gráfico 3: Relação entre o volume de contratos "NonRept_Positions_Short_All" e as cotações do trigo na CBOT (US\$/bushel)

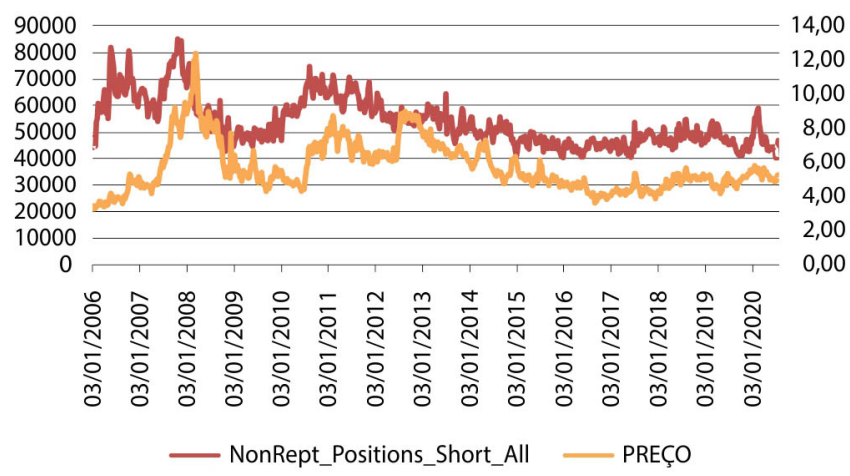

Fonte: elaborado pelos autores, com base nos dados da CBOT e da CFTC.

Gráfico 4: Relação entre o volume de contratos "NComm_Positions_Short_All_NoCIT" e as cotações do trigo na CBOT (US\$/bushel).

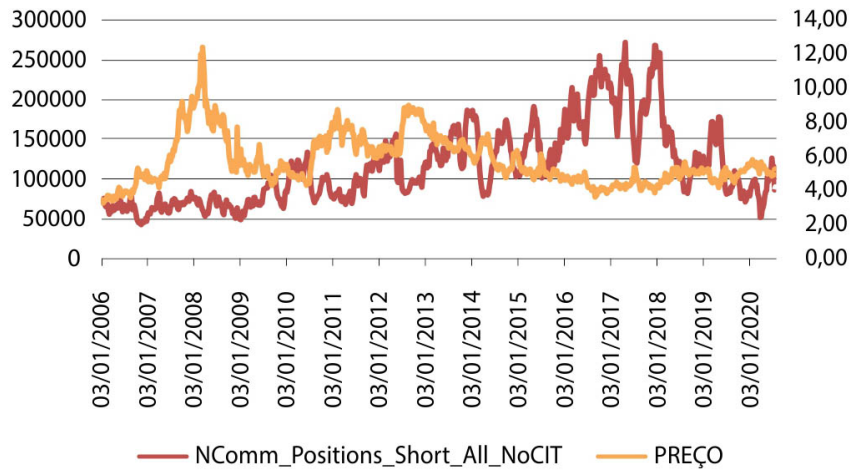

Fonte: elaborado pelos autores, com base nos dados da CBOT e da CFTC

Gráfico 5: Relação entre o volume de contratos "Comm_Positions_Long_All_NoCIT" e as cotações do trigo na CBOT (US\$/bushel).

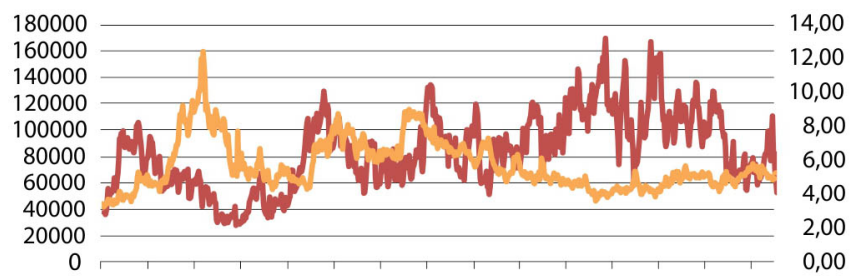

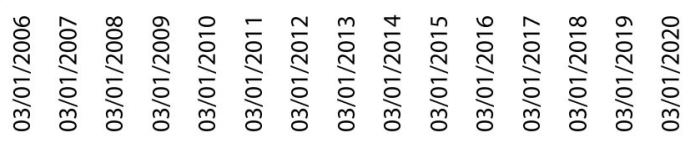

CComm_Positions_Long_All_NoCIT PREÇO

Fonte: elaborado pelos autores, com base nos dados da CBOT e da CFTC. 
Por sua vez, a partir dos modelos de regressão obtidos via stepwise, apresenta-se de que maneira os grupos de investidores explicam a formação do preço futuro do trigo. No quadro 5 se observa, a partir das $\mathrm{R}^{2}$ dos modelos, o poder de explicação de cada um dos cinco modelos propostos. Destaca-se que a variável “CIT_Positions_Long” foi a que apresentou o maior poder de explicação, confirmando o que foi previamente verificado por meio dos índices de correlações. Ela esteve presente em todos os cinco modelos definidos, fato esse que comprova o impacto dessa variável no preço futuro do trigo.

Destaca-se que o melhor modelo explicativo obtido se refere ao quinto modelo, com uma $\mathrm{R}^{2}$ de 0,387 , ou seja, um modelo que explica cerca de $38 \%$ o preço futuro do trigo. Em comparação com outros estudos realizados de formação do preço do grão da soja, do óleo de soja, do farelo de soja e do milho (BRUM, et al. 2019a; BRUM, et al. 2019b; BRUM, et al. 2019c e BRUM, et al. 2020 e SOUZA, et al. 2020), esta commodity foi a que apresentou o mais baixo modelo explicativo. Fato este que comprova que os grupos de investidores impactam na formação do preço futuro do trigo, porém, outras variáveis possuem uma importância ainda mais elevada.

Ao levar em consideração somente a variável "CIT_Positions_Long”, ela sozinha explica cerca de $21 \%$ o preço futuro do trigo e, ao incluir mais quatro variáveis ("Comm_Positions_Long", "Ncomm_Positions_Long", "NonRept_

Quadro 5: Modelos de regressões a partir do método stepwise.

\begin{tabular}{|c|c|c|c|c|c|c|c|}
\hline Modelos & & Beta & $t$ & Sig & Z & Sig. & $\mathbf{R}^{2}$ \\
\hline \multirow{2}{*}{ Modelo 1} & (Constante) & & 8,420 &, 000 & \multirow{2}{*}{203.986} & \multirow{2}{*}{0,000} & \multirow{2}{*}{212} \\
\hline & CIT_Positions_Long_All & ,460 & 14,282 & 000 & & & \\
\hline \multirow{3}{*}{ Modelo 2} & (Constante) & & 10,619 &, 000 & \multirow{3}{*}{126.607} & \multirow{3}{*}{0,000} & \multirow{3}{*}{250} \\
\hline & CIT_Positions_Long_All & 405 & 12,401 &, 000 & & & \\
\hline & Comm_Positions_Long_All_NoClT &,- 204 & $-6,246$ &, 000 & & & \\
\hline \multirow{4}{*}{ Modelo 3} & (Constante) & & 6,677 &, 000 & \multirow{4}{*}{123,060} & \multirow{4}{*}{0,000} & \multirow{4}{*}{328} \\
\hline & CIT_Positions_Long_All & 441 & 14,132 &, 000 & & & \\
\hline & Comm_Positions_Long_All_NoClT &,- 378 & $-10,461$ &, 000 & & & \\
\hline & NComm_Positions_Long_All_NoCIT & & 9,337 &, 000 & & & \\
\hline \multirow{5}{*}{ Modelo 4} & (Constante) & & 4,263 &, 000 & \multirow{5}{*}{103,381} & \multirow{5}{*}{0,000} & \multirow{5}{*}{,354 } \\
\hline & CIT_Positions_Long_All & ,291 & 7,104 &, 000 & & & \\
\hline & Comm_Positions_Long_All_NoCIT &,- 376 & $-10,609$ &, 000 & & & \\
\hline & NComm_Positions_Long_All_NoCIT & 328 & 9,313 &, 000 & & & \\
\hline & NonRept_Positions_Short_All & ,219 & 5,489 &, 000 & & & \\
\hline \multirow{6}{*}{ Modelo 5} & (Constante) & & 3,325 & 001 & \multirow{6}{*}{95,232} & \multirow{6}{*}{0,000} & \multirow{6}{*}{,387 } \\
\hline & CIT_Positions_Long_All & 150 & 3,280 & 001 & & & \\
\hline & Comm_Positions_Long_All_NoClT &,- 429 & $-12,075$ &, 000 & & & \\
\hline & NComm_Positions_Long_All_NoCIT & ,297 & 8,560 & 000 & & & \\
\hline & NonRept_Positions_Short_All & 359 & 8,047 &, 000 & & & \\
\hline & CIT_Positions_Short_All & ,222 & 6,391 & 000 & & & \\
\hline
\end{tabular}

Fonte: resultado da pesquisa com base em dados da CBOT e CFTC. 
Positions_Short"e "CIT_Positions_Short") o poder explicativo do modelo salta para $38,7 \%$.

No momento em que se analisa separadamente somente o grupo de investidores relacionado aos fundos de investimento, os CIT, é possível observar que o $\mathrm{R}^{2}$ do modelo é de 0,214. Além disso, a variável "CIT_Positions_Short” não é significativa a um nível de $5 \%$, portanto, o modelo a seguir demonstra que somente a variável “CIT_Positions_Long” impacta positivamente no preço futuro do trigo e é estatisticamente significativa a um nível de $1 \%$.

Quadro 6: Importância dos CIT's na explicação do preço futuro do trigo.

\begin{tabular}{|l|c|c|c|c|c|c|}
\hline Variáveis & Beta & T & Sig. & Z & Sig. & $\mathbf{R}^{2}$ \\
\hline (Constante) & & 7,860 &, 000 & & & \\
\cline { 1 - 4 } CIT_Positions_Long_All &, 455 & 14,052 &, 000 & 103,118 & 0,000 &, 214 \\
\cline { 1 - 4 } CIT_Positions_Short_All &, 046 & 1,409 &, 159 & & & \\
\hline
\end{tabular}

Fonte: resultado da pesquisa com base em dados da CBOT e CFTC.

Ao agregar os outros grupos de investidores, conhecidos como especuladores, ou seja, os que não precisam declarar as suas informaçóes, e os grupos não comerciais, a $\mathrm{R}^{2}$ do modelo se amplia para um nível de 0,328 , ou seja, cerca de $32 \%$ do preço do trigo pode ser explicado pela atuação dos fundos de investimento, especuladores e negociadores que não precisam declarar as suas negociaçóes, como pode ser observado no quadro a seguir.

Quadro 7: Importância dos CIT's, especuladores e não-comerciais na explicação do preço futuro do trigo.

\begin{tabular}{|c|c|c|c|c|c|c|}
\hline Variáveis & Beta & $\mathrm{t}$ & Sig. & z & Sig. & $\mathbf{R}^{2}$ \\
\hline (Constante) & & 4,788 & ,000 & \multirow{8}{*}{52,604} & \multirow{8}{*}{0,000} & \multirow{8}{*}{328} \\
\hline NComm_Positions_Long_All_NoCIT & 285 & 7,089 & 000 & & & \\
\hline NComm_Positions_Short_All_NoCIT &,- 282 & $-5,585$ &, 000 & & & \\
\hline NComm_Postions_Spread_All_NoCIT &,- 090 & $-1,902$ & 058 & & & \\
\hline NonRept_Positions_Long_All &,- 047 & $-1,029$ & ,304 & & & \\
\hline NonRept_Positions_Short_All & 247 & 4,253 &, 000 & & & \\
\hline CIT_Positions_Long_All & 196 & 3,889 & 000 & & & \\
\hline CIT_Positions_Short_All & 155 & 3,310 & 001 & & & \\
\hline
\end{tabular}

Fonte: resultado da pesquisa com base nos dados da CBOT e da CFTC. 
No momento em que se analisam os grupos de investidores comerciais, formados por "Comm_Positions_Long_All_NoCIT" e "Comm_Positions Short_All_NoCIT", nota-se que eles apresentam uma $\mathrm{R}^{2}$ de $25,2 \%$, valor esse muito próximo dos fundos de investimento.

Quadro 8: Importância dos grupos de investidores comerciais na explicação do preço futuro do trigo.

\begin{tabular}{|l|c|c|c|c|c|c|}
\hline Variáveis & Beta & T & Sig. & Z & Sig. & R $^{2}$ \\
\hline (Constante) & & 21,547 &, 000 & & & \\
\cline { 1 - 4 } Comm_Positions_Long_All_NoCIT &,- 277 & $-8,778$ &, 000 & 127,407 & 0,000 & \multirow{2}{*}{, 252} \\
\cline { 1 - 4 } Comm_Positions_Short_All_NoCIT &, 393 & 12,459 &, 000 & & & \\
\hline
\end{tabular}

Fonte: resultado da pesquisa com base nos dados da CBOT e da CFTC.

Portanto, analisando-se os resultados apresentados, sistematiza-se os mesmos sobre as hipóteses lançadas no quadro 9 a seguir.

Enfim, conforme as hipóteses elencadas para análise e verificação, a hipótese aceita é correspondente apenas a H6: ou seja, os grupos de investidores "CIT long" impactam positivamente no preço futuro do trigo.

Quadro 9: Resultado das hipóteses estudadas.

\begin{tabular}{|c|l|l|}
\hline Número & \multicolumn{1}{|c|}{ Hipótese } & Resultado \\
\hline$H 1:$ & $\begin{array}{l}\text { Os grupos de investidores "Não-Comerciais } \\
\text { short" impactam negativamente no preço } \\
\text { futuro do trigo }\end{array}$ & Rejeitado \\
\hline H2: & $\begin{array}{l}\text { Os grupos de investidores "Não-Comerciais } \\
\text { long" impactam positivamente no preço } \\
\text { futuro do trigo }\end{array}$ & Rejeitado \\
\hline H3: & $\begin{array}{l}\text { Os grupos de investidores "Comerciais long" } \\
\text { impactam positivamente no preço futuro do } \\
\text { trigo }\end{array}$ & Rejeitada \\
\hline$H 4:$ & $\begin{array}{l}\text { Os grupos de investidores "Comerciais short" } \\
\text { impactam negativamente no preço futuro } \\
\text { do trigo }\end{array}$ & Rejeitada \\
\hline H5: & $\begin{array}{l}\text { Os grupos de investidores "CIT short" } \\
\text { impactam negativamente no preço futuro } \\
\text { do trigo }\end{array}$ & Rejeitada \\
\hline
\end{tabular}

Fonte: elaborado pelos autores. 


\section{CONSIDERAÇÕES FINAIS}

À guisa de conclusão, tem-se que o grupo de investidores "CIT Positions Long” tem uma correlação de 0,460 em relação as cotaçóes do trigo em Chicago. Ou seja, tais cotaçóes acompanham o volume de contratos negociados por este grupo de investidores de maneira praticamente direta. $\mathrm{O}$ mesmo pode ser observado com os grupos "NonRept Positions Short All" e "Comm Positions Short All NoCIT”, ambos com correlação respectiva de 0,436 e 0,419.

No que diz respeito aos modelos de regressóes, o estudo demonstrou que o quinto modelo estruturado explica em $38 \%$ o preço futuro do trigo, sendo ele o de maior efeito explicativo. Todavia, em relação a estudos similares feitos com outras commodities, como soja, farelo de soja, óleo de soja e milho, é para o trigo que o modelo em questão apresenta o mais baixo nível de explicação, indicando que há outras variáveis impactando de forma mais relevante o preço do cereal na $C B O T$. Vale ainda destacar que, das variáveis que compóem o quinto modelo, a "CIT Positions Long” sozinha explica $21 \%$ o comportamento das cotaçốes do trigo em Chicago, corroborando sua importância quando na análise das correlaçóes.

Por outro lado, enquanto a variável "CIT Positions Short” não se mostrou significativa, cerca de $32 \%$ do preço do trigo em Chicago seria explicado pela atuação conjunta dos fundos de investimento, dos especuladores e dos chamados negociadores que não precisam declarar as suas negociaçóes.

Quanto a atuação dos investidores comerciais, os mesmos apresentam uma influência de $25 \%$ no comportamento do preço do trigo em Chicago, indicando que os mesmos estão praticamente nos mesmos níveis de influência que os fundos de investimento.

Enfim, os resultados obtidos a partir dos índices de correlaçóes e das regressóes comprovam a aceitação de apenas uma das seis hipóteses lançadas, sendo que as demais foram rejeitadas. Ressalta-se a inversão dos resultados esperados nas hipóteses H3: e H4:, sendo que os grupos de investidores "Comerciais Long" impactaram negativamente e os "Comerciais Short" positivamente no preço futuro do trigo. Portanto, novos estudos poderão ser realizados buscando inserir novas variáveis que elevem o poder explicativo dos modelos e a explicação à formação do preço futuro do trigo. 


\section{Referências}

BAGGIO, D. K.; FERRUZ, L. A.; SARTO, J. L. M. \& KELM, M. L. Los Fondos de Inversión de Brasil: una perspectiva histórica y evolutiva. Revista de Estudos de Administração, v. 5, pp. $53-70,2007$.

BAGGIO, D. K.; FERRUZ, L. A. \& MARCO, I. M. ¿Es el desempeño de los fondos de inversión de Brasil un indicador de movimiento futuro de su patrimonio? El Trimestre Economico, v. 77, n. 306, pp. 445-471, 2010.

BAGGIO, D. K. Los fondos de inversión socialmente responsable de Brasil. Faculdade de Economia e Empresas, Universidad de Zaragoza. Zaragoza (Espanha). Tese de Doutorado, 2012.

BAGGIO, D. K.; SILVEIRA, J. S. T.; SCHNEIDER, I. N.; MACIEL, A. C. \& OLIVA, O. C. Las medidas de performance en la gestión de fondos de inversión socialmente responsables. Revista Academica Contribuciones a la Economia, abril - junho, 2018.

BAGGIO, D. K. et al. Persistência de fundos de investimentos de direito creditório do agronegócio, indústria e comércio. Revista da Universidade Vale do Rio Verde, v. 17, p. 1-9, 2019

BRUM, A.L. et al. A influência dos fundos de investimentos na formação do preço da soja na Bolsa de Cereais de Chicago. 2019a (working paper).

BRUM, A.L. et al. A influência dos fundos de investimentos na formação do preço do farelo de soja na Bolsa de Cereais de Chicago. 2019b (working paper).

BRUM, A.L. et al. A influência dos fundos de investimentos na formação do preço do óleo de soja na Bolsa de Cereais de Chicago. 2019c (working paper)

BRUM, A.L. et al. A influência dos fundos de investimentos na formação do preço do milho na Bolsa de Cereais de Chicago. 2020 (working paper)

BRUM, A.J. A modernização no Planalto Gaúcho: trigo e soja. Ed. Vozes: Petrópolis (RJ), 1988.
CONAB. Disponível em: https://bit.ly/3yerRSo. Acesso em: 15/set./2020.

CORREIA, Linalva Silva; Lima, Sergiany de Silva. Análise da elasticidade de transmissão de preços externos no mercado doméstico da soja maranhense. Revista Desenvolvimento em Debate, Rio de Janeiro/RJ, v. 8, n. 2, maio-agosto de 2020, pp. 141-164.

CALLEGARI-JACQUES, S. M. Bioestatística: princípios e aplicações. Porto Alegre: Artemed, 2003. 255p.

CAMERON, S. Why is the R square Adjusted Reposted? Journal of Quantitative Economics, v.9, n.1, pp. 183-186, 1993.

CBOT. Disponivel em: https://bit.ly/2Wx8 wh7. Acesso em: 02 mar 2020.

CFTC (EUA). Relatório Semanal. Disponível em: https://bit.ly/3ispNgm. Acesso em 10 mar. 2020.

CORRAR, L. J.; PAULO, E. \& DIAS FILHO, J. M. Análise Multivariada para cursos de administração, ciências contábeis e economia. São Paulo: Atlas, 2009.

COUTINHO FILHO, N.R. A geopolítica do petróleo e a influência nos preços das principais commodities agrícolas brasileiras. UFRN, Natal-RN, 2018. 159 p. (dissertação de mestrado)

FERRUZ AGUDO, L. et al. Stochastic frontiers of efficiency for Brazilian investment funds: a panel data analysis. Investment $\mathrm{Ma}$ nagement \& Financial Innovations (print), v. 16, 2019, pp. 352-365.

FIGUEIREDO FILHO, D. B. \& SILVA JÚNIOR, J. A. da. Desvendando os Mistérios do Coeficiente de Correlação de Pearson (r). Revista Política Hoje, v. 18, nl, 2009.

GALVÃO, M.C. et al. Influência do preço mundial do petróleo sobre os preços da soja e do açúcar: uma análise para o Brasil. XLVII SBPO, Porto de Galinhas-PE, 2015, pp. 1193-1204.

HAIR JR, J. F. et al. Análise multivariada de dados. 5. Ed. Porto Alegre: Bookman, 2005. 
HERREROS, M. M. A. G.; BARROS, F. G. N. \& BENTES, E. S. Atividade especulativa dos fundos de investimento no mercado futuro de commodities agrícolas, 2006 - 2009. Revista Política Agrícola, n. 1, jan/fev/mar, 2010.

LIRA, S. A. Análise de correlação: abordagem teórica e de construção dos coeficientes com aplicações. Dissertação apresentada ao Curso de Pós-Graduação em Métodos Numéricos em Engenharia dos Setores de Ciências Exatas e de Tecnologia da Universidade Federal do Paraná, como requisito parcial à obtenção do Grau de Mestre em Ciências. Curitiba, 2004.

LISZBINSKI, B. B. et. al. Sustainability in Soybean Production from the Perspective of the Producers. Journal of Management and Sustainability, v. 10, pp. 138-151, 2020.

MARGARIDO, M.A.; TUROLLA, F.A. \& BUENO, C. R. F. Análise da volatilidade e transmissão de preços entre os mercados internacionais de petróleo e soja. Revista Organizações Rurais \& Agroindustriais, Lavras-MG, v. 16, n. 1, pp. 123-138, 2014.
MONTGOMERY, D. C.; PECK, E. A \& VINING, G. G. Introduction to linear regression analysis, 3nd ed, Wiley-Interscience, 2006.

SCHNEIDER, I. N. et al. Assessing Market Timing Performance of Brazilian Multi-Asset Pension Funds using the Battese and Coelli's Stochastic Frontier Model (1995). Economics Bulletin, v. 40, p. 1-12, 2020.

SELL, I. Utilização da regressão linear como ferramenta de decisão na gestão de custos. In: Anais do IX Congresso Internacional de Custos, pp. 1-16, 2005.

SILVEIRA, D. C. da. et al. As exportações da região Sul do Brasil: uma análise do setor agropecuário. Revista REDES - Santa Cruz do Sul (On_line). Santa Cruz do Sul/RS, v. 24, n. 3, pp. 272-294, setembro-dezembro, 2019. SOUZA, F. M. The establishment of soybean price and its interrelation with the prices of its derivates: oil and meal. 2020 (working paper).

USDA. World Agricultural Supply and Demand Estimates. Washington DC, USA, 11 set. 2020.40 p.

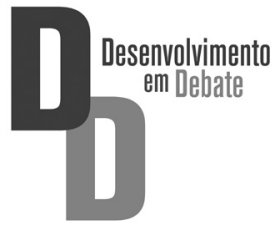

